\title{
The cumulative amount of serum-free light chain is a strong prognosticator in chronic lymphocytic leukemia
}

\author{
Fortunato Morabito, ${ }^{1}$ Rosaria De Filippi, ${ }^{2,3}$ Luca Laurenti, ${ }^{4}$ Katja Zirlik, ${ }^{5}$ Anna Grazia Recchia, ${ }^{1}$ Massimo Gentile,${ }^{1}$ \\ Emanuela Morelli, ${ }^{2}$ Ernesto Vigna, ${ }^{1}$ Vincenzo Gigliotti, ${ }^{1}$ Rosa Calemma, ${ }^{6}$ Barbara Amoroso, ${ }^{7}$ Antonino Neri, ${ }^{8}$ \\ Giovanna Cutrona, ${ }^{9}$ Manlio Ferrarini, ${ }^{9}$ Stefano Molica, ${ }^{10}$ Giovanni Del Poeta, ${ }^{11}$ Claudio Tripodo, ${ }^{12}$ and Antonio Pinto ${ }^{2}$

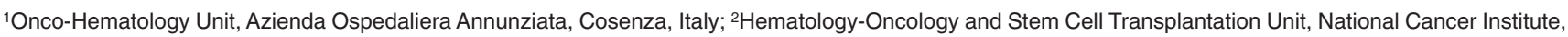 \\ Fondazione G. Pascale, Istituto di Ricovero e Cura a Carattere Scientifico (IRCCS), Naples, Italy; ${ }^{3}$ Department of Cellular and Molecular Biology and Pathology, \\ Faculty of Biotechnological Sciences, Federico II University, Naples, Italy; ${ }^{4}$ Department of Hematology, Catholic University Sacro Cuore, A. Gemelli Hospital, \\ Rome, Italy; ${ }^{5} \mathrm{Hematology/Oncology,} \mathrm{University} \mathrm{Medical} \mathrm{Center} \mathrm{Freiburg,} \mathrm{Freiburg,} \mathrm{Germany;}{ }^{6}$ Oncological Immunology, National Cancer Institute, Naples, \\ G. Pascale, IRCCS, Naples, Italy; ${ }^{7}$ Clinical Research Direction, The Binding Site, Rome, Italy; ${ }^{8}$ Centro di Ricerca per lo Studio delle Leucemie, Fondazione \\ IRCCS, Università di Milano, Milano, Italy; ' Divisione di Oncologia Medica C, Istituto Nazionale per la Ricerca sul Cancro, Istituto Tumori, Genova, Italy; \\ ${ }^{10}$ Oncologia Medica, Azienda Ospedaliera Pugliese Ciaccio, Catanzaro, Italy; ${ }^{11}$ Division of Hematology, S. Eugenio Hospital and University of Tor Vergata, \\ Rome, Italy; and ${ }^{12}$ Department of Health Sciences, Human Pathology Section, University of Palermo, Palermo, Italy
}

Identification of patients at risk of early disease progression is the mainstay of tailored management in chronic lymphocytic leukemia (CLL). Although application of established biomarkers is limited by intrinsic detection/readout complexities, abnormality of $\kappa$ and $\lambda$ serum-free light chain ratio [sFLC $(\kappa / \lambda)$ ] was proposed as a straightforward prognosticator in CLL. By analyzing 449 therapynaive patients, we show that an abnormal $\operatorname{sFLC}(\kappa / \lambda)$, along with CD38, ZAP-70, IGHV mutations, cytogenetics and stage, inde- pendently predicts treatment-free survival (TFS) but becomes prognostically irrelevant if the cumulative amount of clonal and nonclonal FLCs [sFLC $(\kappa+\lambda)$ ], a variable associated with cytogenetic risk, exceeds the threshold of $60.6 \mathrm{mg} /$ $\mathrm{mL}$. Patients with sFLC $(\kappa+\lambda)$ above cutoff displayed a poorer TFS outcome, irrespective of $s F L C(\kappa / \lambda)$. Only ZAP-70, cytogenetics, stage, and TFS remained associated with SFLC $(\kappa+\lambda)$ in a multivariate model. By assigning 1 point each for these variables, the 3-year probability of
TFS was $94.8 \%, 84.5 \%, 61.6 \%$, and $21.1 \%$ for patients scoring $0,1,2$, and $3+4$, respectively $(P<.0001)$. These data, and the demonstration that monoclonal and polyclonal B cells concur to FLC synthesis in tumor tissues, suggest that $\operatorname{sFLC}(\kappa / \lambda)$ and $\operatorname{sFLC}(\kappa+\lambda)$ mirror distinct biologic processes in CLL. $\operatorname{sFLC}(\kappa+\lambda)$ assessment represents a sensitive and cost-effective tool for identifying CLL patients requiring early treatment. (Blood. 2011;118(24):6353-6361)

\section{Introduction}

During physiologic lymphopoiesis, B cells and plasma cells mostly produce an intact immunoglobulin ( $\mathrm{Ig}$ ), consisting of heavy chains bound to a $\kappa$ - or $\lambda$-light chain, together with an excess, approximately $40 \%$, of unbound free light chains (FLCs). ${ }^{1}$ In plasma cell malignancies, such as multiple myeloma (MM) and related disorders, tumor cells usually secrete intact monoclonal Igs along with a relative excess of clonally related FLCs, whereas, in rare cases, only a clonal light chain without identifiable heavy chain is produced. ${ }^{1}$ A straightforward nephelometric assay, the serum FLC (sFLC) assay, has been developed that accurately measures the amounts of free $\mathrm{\kappa}$ - and $\lambda$-light chains circulating in the serum as monomers or dimers unbound to the Ig heavy chains. ${ }^{2}$ The assay allows the detection of abnormalities in the physiologic ratios of sFLCs $[\mathrm{sFLC}(\kappa / \lambda)]$, which can be used to reveal the presence of clonal, $\kappa$ - or $\lambda$-restricted tumor cell populations, and permits quantization of sFLC absolute levels, which correlate with disease activity and clinical outcome in patients with plasma cell malignancies. $^{3}$ In addition, sFLC elevations, with a conserved $\operatorname{sFLC}(\kappa / \lambda)$, were shown to represent a tool to quantify ongoing polyclonal B-cell activation/expansions, underlying the pathogenesis of vari-

Submitted April 26, 2011; accepted September 20, 2011. Prepublished online as Blood First Edition paper, October 13, 2011; DOI 10.1182/blood-2011-04345587.

The online version of this article contains a data supplement. ous chronic inflammatory and autoimmune conditions, ${ }^{4}$ as well as to be an indirect way to measure progressive renal injury, which is characterized by an impaired FLC catabolism. ${ }^{5}$

Although the relative ratio of intact Ig to FLC production in B-cell malignancies other than MM has been relatively less explored, early studies indicated that in chronic lymphocytic leukemia (CLL) and some B-cell non-Hodgkin lymphomas, tumor cells may display a significant imbalance in heavy and light chain synthesis leading to the secretion of a large excess of FLCs compared with heavy chains and intact Igs. ${ }^{6}$ Being a sensitive, simple, and reproducible indicator of B-cell clonality and biologic activity, the $\operatorname{sFLC}(\kappa / \lambda)$ test is particularly attractive as a putative prognostic marker in CLL, ${ }^{7,8}$ a B-cell tumor whose clinical course is highly heterogeneous across individual patients. Many patients are asymptomatic and survive for decades without requiring treatment, whereas others experience an aggressive form of disease and may die of disease- or therapy-related complications shortly after diagnosis. Within such a clinical framework, biologic predictors of clinical progression would be of great value, at diagnosis, to identify patients at risk of requiring early treatment and spare the marked "advertisement" in accordance with 18 USC section 1734.

(c) 2011 by The American Society of Hematology 
toxic load of unnecessary therapy to those predicted to display an indolent course.

A number of biomarkers, including ZAP-70, CD38, immunoglobulin heavy chain variable gene (IGHV) mutation status, and cytogenetic abnormalities, have been validated that rank the risk of progressive disease and predict treatment requirement and survival in the individual CLL patient. ${ }^{9}$ However, assessment methodologies, being complex and expensive, are not readily available in all instances, mostly limiting application of these biomarkers to the context of clinical trials and specific patient situations, also because of reproducibility and standardization issues across laboratories.

Two previous reports have indicated that $\operatorname{sFLC}(\kappa / \lambda)$ abnormalities are present in a significant proportion of CLL patients and could independently identify those at risk of progressive disease. ${ }^{7,8}$ These retrospective studies, however, assessed small cohorts of both therapy-naive and treated patients, leading to varied results as to the prognostic value of $\operatorname{sFLC}(\kappa / \lambda)$, rather than absolute sFLC levels, and the predictive power of sFLC abnormalities within the framework of other CLL biomarkers.

By analyzing the largest cohort of therapy-naive CLL patients so far reported, we wished to: (1) determine the independent predictive value of sFLC abnormalities in the context of established CLL biomarkers; (2) define the predictive hierarchy of these markers; (3) dissect the prognostic relevance of $\operatorname{sFLC}(\kappa / \lambda)$ from the predictive value of sFLC absolute levels; and (4) incorporate sFLC-dependent variables into a novel prognostic system able to predict treatment-free survival (TFS) in newly diagnosed patients.

\section{Methods}

\section{Patients and study design}

A total of 449 previously untreated CLL patients from a cooperative network of Italian institutions were enrolled in a collaborative study. All participants provided written informed consent in accordance with the Declaration of Helsinki, and the study was approved by the relevant institutional review boards. Inclusion criteria consisted of a diagnosis of typical CLL based on National Cancer Institute, Working Group criteria ${ }^{10}$ and confirmed by flow cytometry. ${ }^{11-13}$ Availability of serum samples obtained within 3 months from initial diagnosis in the absence of any therapeutic intervention, including low-dose steroids and anti-inflammatory treatments, was required at study entry. Table 1 summarizes the main clinical and biologic data from this group. The median follow-up was 3.0 years, and 149 of 449 patients analyzed for clinical outcome had received treatment by the end of the study. TFS was measured from diagnosis to first-line treatment or last follow-up. Treatment was decided uniformly in all participating centers based on documented progressive and symptomatic disease according to National Cancer Institute, Working Guidelines. ${ }^{10}$

\section{CD38 and ZAP-70 determination, IGHV gene analysis, and cytogenetics}

All flow cytometric analyses reported in this study were performed on a FACSCalibur flow cytometer (BD Biosciences). The expression of CD38 was analyzed by 3 -color immunofluorescence, ${ }^{12,14}$ and the detection of ZAP-70 was performed according to previously reported methods. ${ }^{12,14}$ The cut-off of $30 \%$ positive cells was chosen to discriminate $\mathrm{CD}^{-} 8^{-}$from $\mathrm{CD}^{3} 8^{+}$CLL. ${ }^{14}$ The cut-off levels of $20 \%{ }^{15}$ or $30 \%^{16}$ were used to distinguish $\mathrm{ZAP}_{-} 70^{-}$from ZAP-70 ${ }^{+}$CLL, depending on anti-ZAP-70 antibody used and on each laboratory's standardization of ZAP-70 flow cytometric protocols.

$I G H V$ gene usage and mutation status were determined using cDNA according to previously published methods. ${ }^{17}$
Table 1. Clinical, biologic, and molecular features of CLL patients included in the study

\begin{tabular}{lc}
\hline Parameter & No. (\%) of patients \\
\hline Total patients & 449 \\
Age, $\mathbf{y}$ & $449(100)$ \\
Median & 65 \\
\hline Range & $33-89$ \\
Sex & $449(100)$ \\
\hline Female & $167(37.2)$ \\
\hline Male & $282(62.8)$ \\
\hline Binet stage & $449(100)$ \\
\hline A & $331(73.7)$ \\
\hline B & $67(14.9)$ \\
C & $51(11.4)$ \\
\hline ZAP-70 expression & $401(89.3)$ \\
\hline Negative & $261(65.1)$ \\
\hline Positive & $140(34.9)$ \\
CD38 expression & $410(91.3)$ \\
\hline Negative & $330(80.5)$ \\
\hline Positive & $80(19.5)$ \\
\hline GHV mutational status & $379(84.4)$ \\
\hline Mutated & $249(65.7)$ \\
\hline Unmutated & $130(34.3)$ \\
\hline FISH analysis & $303(67.5)$ \\
\hline Normal & $135(44.6)$ \\
\hline 13q14.3 & $83(27.4)$ \\
\hline Trisomy 12 & $32(10.6)$ \\
\hline 11q22.3 & $29(9.6)$ \\
\hline 17p13.1 & $24(7.9)$ \\
\hline & \\
\hline
\end{tabular}

Interphase FISHs were carried out for the detection of trisomy 12 and chromosome deletion at 17p13.1, 11q22.3, and 13q14.3 loci. Dual-color hybridizations using appropriate centromeric-specific probes and unique sequence-specific probe for TP53 (LSI P53) and ATM (LSI ATM) loci, were performed for the $17 \mathrm{p} 13.1$ and 11q23.3 deletions, respectively. For the detection of 13q14.3 deletion, the locus-specific probe (LSI D13D25) was cohybridized with the $13 \mathrm{q} 34$ telomeric probe as an internal control for nullisomy. A chromosome 12 -specific $\alpha$-satellite probe was used to identify trisomy 12. All probes were purchased from Vysis, and FISH procedures were performed following the manufacturer's specifications. For each hybridization, a minimum of 200 interphase nuclei were assessed. ${ }^{18}$ Patients were categorized into high (17p13.1 and 11q22.3 deletions), intermediate (trisomy 12), and low (13q14.3 deletion and normal) risk groups for subsequent analysis.

\section{sFLC determination}

Levels of sFLC were determined in all study samples using a particle enhanced, high-specificity, homogeneous immuno-FLC assay (Freelite; The Binding Site) performed on a Delta Nephelometer (Radim Diagnostics). It consists of 2 separate measurements: the first to detect free- $\mathrm{KIg}$ (normal range, $3.3-19.4 \mathrm{mg} / \mathrm{L}$ ) and the other to detect free- $\lambda$ Ig (normal range, $5.7-26.3 \mathrm{mg} / \mathrm{L}$ ) light chains. In addition to measuring absolute sFLC protein levels, the test also allowed us to assess the presence or absence of clonality based on the ratio of free- $\kappa$ to $-\lambda$ Ig concentrations (normal range, $0.26-1.65)$. We adjusted the reference ratios for normality of sFLC $(0.37-3.1 \mathrm{mg} /$ L), as suggested by Hutchison et al, ${ }^{5}$ to reflect progressive changes in renal function in patients with abnormal creatinine clearance. Finally, we introduced a new variable (ie, the total amount of $\kappa$ and $\lambda$ light chains $[\operatorname{sFLC}(\kappa+\lambda)])$ determined by summing the free- $\kappa$ plus free- $\lambda$ chains.

In situ immunohistochemistry was performed on formalin-fixed, paraffinembedded lymph node and bone marrow samples relative to 4 CLL and $2 \mathrm{MM}$ cases as previously reported. ${ }^{19}$ Briefly, tissue sections were deparaffinized with xylene and rehydrated with water through a graded alcohol series. Slides were microwave treated in citrate buffer, $\mathrm{pH} 6.0$ (Dako North America) and, after Fc blocking with a specific blocking buffer (Protein Block), incubated with the validated primary mouse monoclonal 
Table 2. sFLC ratio abnormality in relation with different biologic and clinical markers in CLL patients

\begin{tabular}{|c|c|c|c|c|}
\hline & \multicolumn{4}{|c|}{ sFLC } \\
\hline & All patients, no. (\%) & Normal, no. (\%) & Abnormal, no. (\%) & $P$ \\
\hline CD38 & $410(100)$ & $270(65.9)$ & $140(34.1)$ & \\
\hline Negative & $330(80.5)$ & $234(70.9)$ & $96(29.1)$ & $<.0001^{*}$ \\
\hline Positive & $80(19.5)$ & $36(45.0)$ & $44(55.0)$ & \\
\hline ZAP-70 & $401(100)$ & $267(66.6)$ & $134(33.4)$ & \\
\hline Negative & $261(65.1)$ & $191(73.2)$ & $70(26.8)$ & $<.0001 \dagger$ \\
\hline Positive & $140(34.9)$ & $76(54.3)$ & $64(45.7)$ & \\
\hline IGHV status & $379(100)$ & $257(67.8)$ & $122(32.2)$ & \\
\hline Mutated & $249(65.7)$ & $182(73.1)$ & 67 (26.9) & $.003^{*}$ \\
\hline Unmutated & $130(34.3)$ & $75(57.7)$ & $55(42.3)$ & \\
\hline Binet stage & $449(100)$ & $299(66.6)$ & $150(33.4)$ & \\
\hline A & $331(80.5)$ & $232(70.1)$ & 99 (29.9) & $.012^{*}$ \\
\hline$B+C$ & $118(19.5)$ & $67(56.8)$ & $51(43.2)$ & \\
\hline FISH & $303(100)$ & $205(67.7)$ & $98(32.3)$ & \\
\hline $13 q 14.3$ and normal (low risk) & $208(71.9)$ & $154(70.6)$ & $64(29.4)$ & \\
\hline Trisomy 12 (intermediate risk) & $32(10.6)$ & $19(59.4)$ & $13(40.6)$ & $.20 \dagger$ \\
\hline $11 \mathrm{q} 22.3$ and $17 \mathrm{p} 13.1$ (high risk) & $53(17.5)$ & $32(60.4)$ & $21(39.6)$ & \\
\hline
\end{tabular}

${ }^{*}$ Fisher Exact Test.

†Pearson $\chi^{2}$ text.

antibodies specific for human $\kappa$ and $\lambda$ FLCs (kindly provided by Dr A. Solomon, Knoxville, TN), whose binding was revealed by LSAB + System-HRP (Dako North America) and 3-3'-diaminobenzidine (Novocastra) substrate-chromogen. Stained slides were counterstained with hematoxylin and captured by a Leica DM2000 optical microscope (Leica Microsystems). Microphotographs were acquired using a Leica DFC320 digital camera and imported using Leica IM50 Version 4.0 imaging software (Leica Microsystems) fitted with a Leica 40×/0.65 HI PLAN lens and IM50 acquisition software (Version 4.0).

\section{Statistical analysis}

The statistical package SPSS for Windows release 13.0 2004 software (SPSS) was used for all analyses. For categorical variables, statistical comparisons were performed using 2-way tables for the Fisher exact test and multiway tables for the Pearson $\chi^{2}$ test. Receiver operating characteristic curve analysis was used to determine the best $\operatorname{sFLC}(\kappa+\lambda)$ cut-off value that could discriminate patients who were actually treated from those who never received therapy at the longest follow-up. TFS analyses were performed using the Kaplan-Meier method. Statistical significance of associations between individual variables and survival was calculated using the log-rank test. The prognostic impact for the outcome variable was investigated by univariate and multiple Cox regression analysis. Data are expressed as hazard ratio (HR) and 95\% confidence interval (CI). Coxderived estimated survival curves according to the combination of prognostic factors were also constructed by the standard approach. ${ }^{20}$ Because HR calculates the magnitude of risk rather than the model's capacity to accurately classify patient outcome, Harrell C-statistics were used to further evaluate the discriminatory power of variables in terms of TFS. ${ }^{21} \mathrm{~A}$ value of $P<.05$ was considered significant for all statistical calculations.

\section{Results}

\section{Assessment of biologic associations and predictive value of $\operatorname{sFLC}(\kappa / \lambda)$}

After correction for renal impairment, an abnormal sFLC $(\kappa / \lambda)$ was found in 150 of 449 cases (33.4\%) and displayed a statistically significant correlation with expression of CD38 and ZAP-70, presence of unmutated $I G H V$, and advanced stage (Table 2); of note, $\operatorname{sFLC}(\kappa / \lambda)$ failed to correlate with cytogenetic abnormalities. At a median follow-up of 3 years, 149 of 449 patients had received treatment. To verify for the potential prognostic relevance of
$\operatorname{sFLC}(\kappa / \lambda)$, patients were divided into 3 groups based on their normal or abnormal $\kappa / \lambda$ ratio (clonal $\kappa$ or clonal $\lambda$ cases). The 3 -year probability of remaining treatment-free for patients with abnormal $\kappa$ or $\lambda \operatorname{sFLC}(\kappa / \lambda)$ was significantly lower than those with a normal $\mathrm{sFLC}(\kappa / \lambda)\left(63.8 \%\right.$ vs $70.1 \%$ vs $84 \%, \chi^{2}$ of log-rank 34 , $P<.0001$; Figure 1). As shown by univariate Cox analysis, advanced stage (Binet B and C) patients (118 of 449; HR = 2.5, 95\% CI, 1.8-3.5, $P<.0001$ ), those expressing CD38 (80 of 410; $\mathrm{HR}=2.7,95 \% \mathrm{CI}, 1.9-3.9, P<.0001)$ or ZAP-70 (140 of 401; $\mathrm{HR}=4.4,95 \% \mathrm{CI}, 3.1-6.3, P<.0001)$, those with unmutated IGHV (130 of 379; HR $=4.1,95 \% \mathrm{CI}, 2.8-6.0, P<.0001)$, and patients with abnormal $\operatorname{sFLC}(\kappa / \lambda)(\mathrm{HR}=2.5,95 \% \mathrm{CI}, 1.8-3.4$, $P<.0001)$ had a statistically significant higher risk of starting treatment (supplemental Figure 1A, available on the Blood Web site; see the Supplemental Materials link at the top of the online

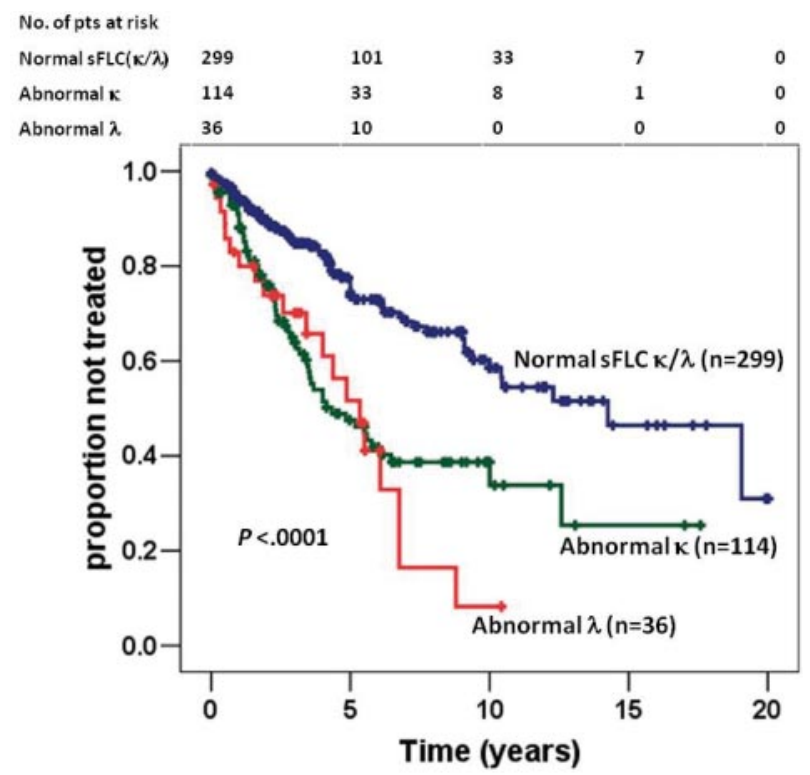

Figure 1. TFS of 449 patients with CLL according to the sFLC assay. KaplanMeier curves show the 3-year probability of remaining treatment-free for CLL cases subdivided into 3 groups based on their normal [normal sFLC $(\kappa / \lambda)$ ] or abnormal $\kappa$ or $\lambda$ sFLC ratio. The 3-year TFS was significantly lower in patients with $\kappa$ or $\lambda \operatorname{sFLC}(\kappa / \lambda)$. 
article). Notably, abnormal sFLC $(\kappa / \lambda)(\mathrm{HR}=2.2,95 \%$ CI, 1.5-3.2, $P<.0001)$ retained an independent association with TFS even when adjusted for CD38, ZAP-70 expression, IGHV mutational status, and Binet stage. ZAP-70 (HR $=3.0,95 \%$ CI, 1.9-4.8, $P<.0001)$, unmutated IGHV $(\mathrm{HR}=1.9,95 \%$ CI, 1.1-3.2, $P=.014)$, and Binet stage $(\mathrm{HR}=2.4,95 \% \mathrm{CI}, 1.6-3.7, P<.0001)$ remained independently associated with the risk of requiring therapy, whereas CD38 expression was no longer predictive (supplemental Figure 1B).

\section{Assessment of the predictive value of $\operatorname{sFLC}(\kappa+\lambda)$}

To further dissect the potential predictive value of sFLC, the $\mathrm{\kappa}$ - and $\lambda$-FLC concentrations were summed to produce a new variable, named sFLC $(\kappa+\lambda)$ (median, $39.0 \mathrm{mg} / \mathrm{mL}$; range, 8.2-1430.4 mg/ $\mathrm{mL}$, supplemental Figure 2A). Because a significantly higher sFLC $(\kappa+\lambda)$ was observed in CLL patients who had required treatment (supplemental Figure 2B), a cut-off value was established to resolve the power of $\operatorname{sFLC}(\kappa+\lambda)$ of identifying patients who were actually treated from those who remained treatment-free at the longest follow-up. Receiver operating characteristic analysis disclosed that $60.6 \mathrm{mg} / \mathrm{mL}$ was the most suitable $\operatorname{sFLC}(\kappa+\lambda)$ cut-off value (area under the curve $=.62, P<.0001$ ). At 3 years, the probability of being therapy-free was $84.1 \%$ and $51.8 \%$ for patients with a $\operatorname{sFLC}(\kappa+\lambda)$ value below and equal $(\mathrm{n}=365)$ or above $(\mathrm{n}=84)$ the cut-off value, respectively ( $\chi^{2}$ of log-rank 85.6, $P<.0001$, Figure 2A). Interestingly, Cox multivariate analysis suggested that the independent predictive value of $\operatorname{sFLC}(\kappa+\lambda)$ above the cut-off ( $\mathrm{HR}=2.5, \mathrm{CI}, 2.6-3.9)$ remained one of the strongest predictive parameters together with ZAP-70 $(\mathrm{HR}=2.8$, $\mathrm{CI}, 1.8-4.6)$ and Binet stage ( $\mathrm{HR}=2.5, \mathrm{CI}, 1.6-3.8)$, whereas $\mathrm{CD} 38$ as well as $I G H V$ mutational status lost their prognostic power.

\section{Relationship between $\operatorname{sFLC}(\kappa+\lambda)$ and $\operatorname{sFLC}(\kappa / \lambda)$}

Because each of the 2 patient cohorts defined by the presence of a summated FLC value below or above the cut-off (Figure 2A) derived from the concurrence of cases with an excess monoclonal sFLC production (ie, with an abnormal sFLC ratio) and cases with an excess polyclonal sFLC production (ie, with a conserved sFLC ratio), we considered it appropriate to further dissect these cohorts. To this end, we proceeded to analyze separately the outcome of patients with both normal sFLC $(\kappa / \lambda)$ and $\operatorname{sFLC}(\kappa+\lambda)$ below the cut-off $(277$ of $449,61.7 \%)$ or both abnormal $\operatorname{sFLC}(\kappa / \lambda)$ and $\operatorname{sFLC}(\kappa+\lambda)$ above the cut-off (62 of 449, 13.8\%), defined as "concordant cases" $(75.5 \%)$, and the outcome of patients with either normal sFLC $(\kappa / \lambda)$ and $\operatorname{sFLC}(\kappa+\lambda)$ above the cut-off ( 22 of $110,20 \%)$ or abnormal $\operatorname{sFLC}(\kappa / \lambda)$ and $\operatorname{sFLC}(\kappa+\lambda)$ below the cut-off ( 88 of 110, 80\%), defined as "discordant cases" (110 of 440, $24.5 \%$; Figure 2B). The estimated Kaplan-Meier curves for TFS according to the combination of $\operatorname{sFLC}(\kappa / \lambda)$ abnormality and $\operatorname{sFLC}(\kappa+\lambda)$ cut-off values are shown in Figure $2 \mathrm{C}$. To better illustrate the predictive impact of summated sFLC on TFS, separate curves for patients with normal (Figure 2D) or abnormal sFLC ratio (Figure $2 \mathrm{E})$ are shown with respect to the $\operatorname{sFLC}(\kappa+\lambda)$ cut-off. Notably, a Cox multivariate model showed that $\operatorname{sFLC}(\kappa+\lambda)$ above cut-off yields an approximately 3 -fold higher risk of early treatment requirement than abnormal $\mathrm{sFLC}(\kappa / \lambda)$ : $\mathrm{HR}=3.5, \mathrm{CI}$, 2.4-5.1 $(P<.0001)$ versus $\mathrm{HR}=1.5, \mathrm{CI}, 1.1-2.3(P=.015)$.

To investigate which FLC-related parameter could provide a more accurate prediction of TFS, a c-statistic analysis, considered a measure of concordance between observed and predicted timedependent events, was carried out. The results demonstrated that both the $\operatorname{sFLC}(\kappa / \lambda)(\mathrm{c}=.51, P<.001)$ and the $\operatorname{sFLC}(\kappa+\lambda)$ (c $=.52, P<.001)$ were able to correctly predict TFS.

\section{Distribution of cytogenetic abnormalities among $\operatorname{sFLC}(\kappa+\lambda)$ and $\operatorname{sFLC}(\kappa / \lambda)$}

A total of 303 samples were investigated by interphase FISH for the presence of the major genomic aberrations. The overall incidence was as follows: (1) 13q14.3 deletion, 83 of 303 (27.4\%) and normal in 135 of 303 (overall 218, 44.6\%, low risk group); (2) trisomy 12, 32 of 303 (10.6\%, intermediate risk group); and (3) 11q22.3 deletion, 29 of $303(9.6 \%)$ and $17 \mathrm{p} 13.1$ deletion 24 of 303 (7.9\%, high risk group). Interestingly, a statistically significant association was documented for risk as defined by FISH analysis and $\operatorname{sFLC}(\kappa+\lambda)(P=.013$, Figure $3 \mathrm{~A})$, but not with $\mathrm{SFLC}(\kappa / \lambda)$ values.

Supplemental Figure 3 shows the Kaplan-Meier curves of cases stratified by cytogenetic risk. The estimated median times to treatment need were 12.1, 6.5, and 3.1 years for cases with low, intermediate, and high risk, respectively. Notably, abnormal $\operatorname{sFLC}(\kappa / \lambda)(\mathrm{HR}=2.0,95 \% \mathrm{CI}, 1.3-3.1)$ and $\operatorname{sFLC}(\kappa+\lambda)$ above the cut-off $(\mathrm{HR}=2.8,95 \% \mathrm{CI}, 1.7-4.5)$ retained an independent association with TFS together with cytogenetics, ZAP-70 expression, and Binet stage (Figure 3B-C). Finally, results from a multivariate model in which both $\operatorname{sFLC}(\kappa+\lambda)$ and $\operatorname{sFLC}(\kappa / \lambda)$ parameters were considered together showed that the latter failed to retain an independent association with TFS, whereas $\operatorname{sFLC}(\kappa+\lambda)$ $(\mathrm{HR}=2.3,95 \% \mathrm{CI}, 1.3-4.1, P=.004)$ still remained a significant prognostic factor together with cytogenetics $(\mathrm{HR}=2.1,95 \% \mathrm{CI}$, $1.5-2.7, P<.0001)$, ZAP-70 $(\mathrm{HR}=2.8,95 \%$ CI, 1.7-4.8, $P<.0001)$ expression, and Binet stage $(\mathrm{HR}=2.8,95 \% \mathrm{CI}$, 1.7-4.6, $P<.0001)$.

\section{A novel prognostic scoring system based on the integration $\operatorname{sFLC}(\kappa+\lambda)$ with 3 biomarkers}

Based on the results of multivariate and c-statistic analyses, we wished to test the hypothesis that the combination of $\operatorname{sFLC}(\kappa+\lambda)$, ZAP-70 expression, and FISH abnormalities together with Binet stage could allow a more finely tuned estimation of prognosis. To this end, we divided cases of our study cohort into 5 groups according to the negative prognostic impact of these biomarkers. A scoring system was then designed in which 1 point was assigned to each unfavorable prognostic marker and the final score was given by the sum of the unfavorable markers present. Based on this analysis of 262 patients, 104 cases scored 0 (low risk), 79 scored 1 (low-intermediate risk), 56 scored 2 (intermediate-high risk), 19 scored 3 , and only 4 cases scored 4 . Considering the relatively low number of cases in the latter 2 score groups, we combined them to form the high-risk group (23 patients). According to this classification, the 3-year probability of avoiding treatment was $94.8 \%$, $84.5 \%, 61.6 \%$, and $21.1 \%$, respectively, for patients in the low, low-intermediate, intermediate-high, and high risk groups (Figure 4).

\section{In situ immunohistochemical detection of $\kappa$ and $\lambda$ FLCs in the CLL microenvironment}

After analysis of the prognostic significance of $\operatorname{sFLC}(\kappa+\lambda)$, we investigated the actual presence and distribution of FLCs in CLL infiltrates in situ, by immunohistochemistry using 2 validated monoclonal antibodies specifically recognizing the unbound light chains. ${ }^{21}$ Consistent with the hypothesized concurrence of different B-cell populations of FLC synthesis in the CLL microenvironment, we detected, along with a prevalent population of plasmacytoid 
A

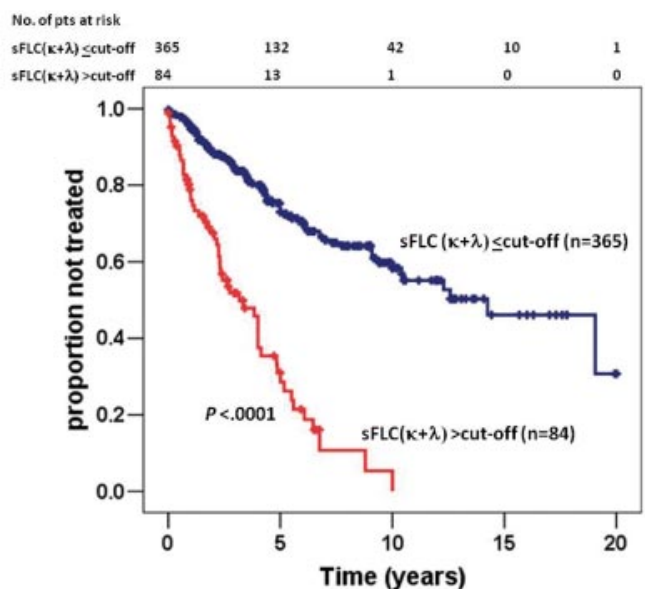

B

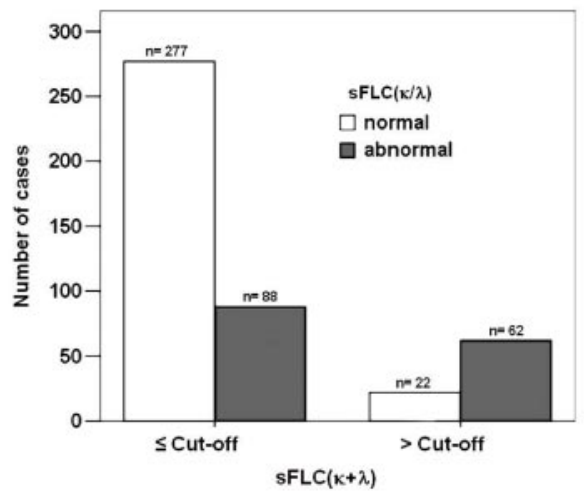

C No.ofts atrisk
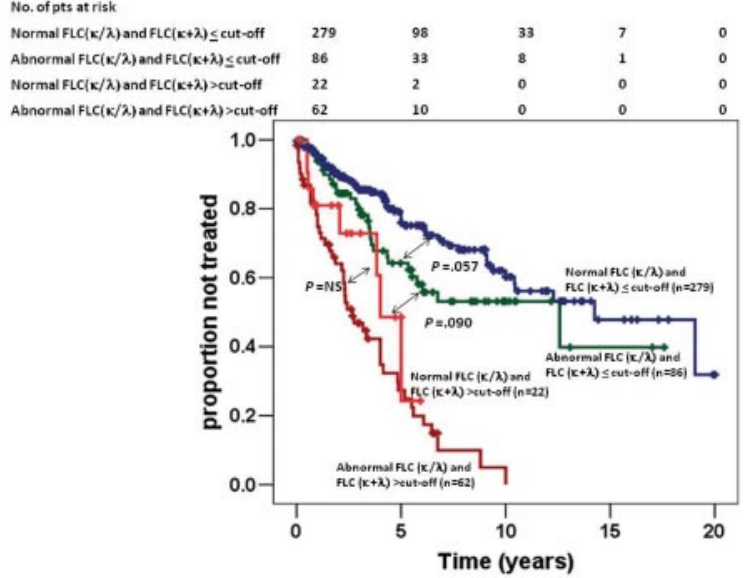

No. of pts atrist

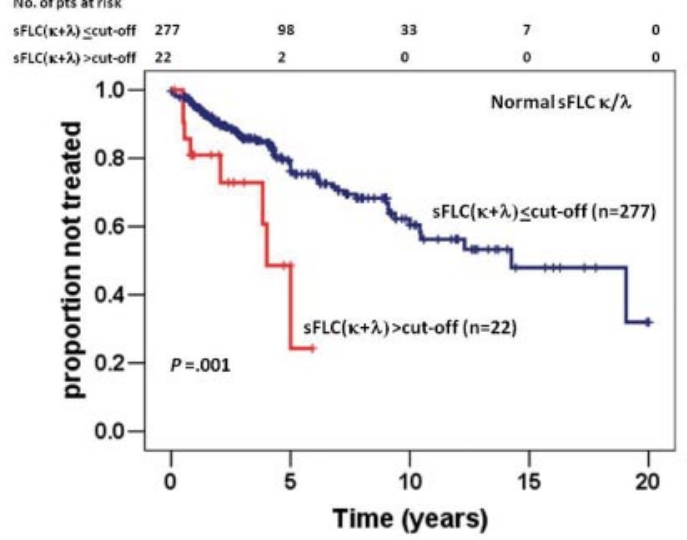

E Nofptsat tisk

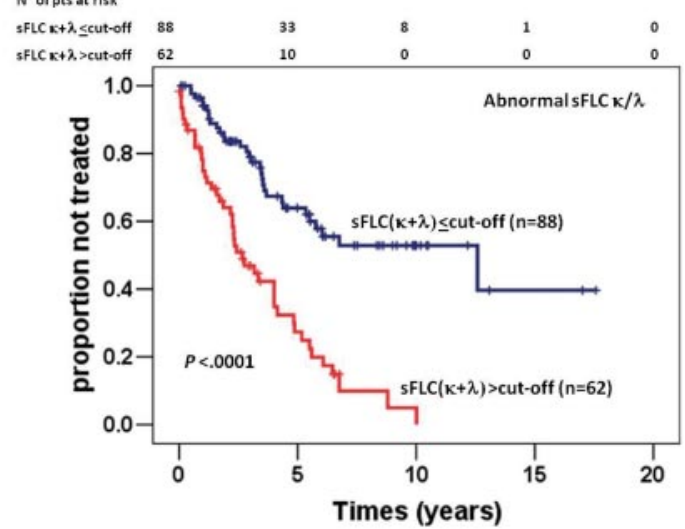

Figure 2. Predictive value of a novel parameter $\operatorname{sFLC}(\kappa+\lambda)$ and relationship with $\operatorname{sFLC}(\kappa / \lambda)$ ratio. (A) Kaplan-Meier curves demonstrating a significantly shorter 3-year TFS for those CLL cases with $\mathrm{SFLC}(\kappa+\lambda)$ reaching or exceeding the cut-off value $\left(51.8 \%, \chi^{2}\right.$ of log-rank $\left.85.6, P<.0001\right)$. (B) Comparison of CLL cases in terms of $\mathrm{sFLC}(\kappa / \lambda)$ and sFLC $(\kappa+\lambda)$ variables with respect to $\mathrm{sFLC}(\kappa+\lambda)$ cut-off value of $60.6 \mathrm{mg} / \mathrm{mL}$. Of a total of 449 cases, $277(61.7 \%)$ had normal sFLC $(\kappa / \lambda)$ and sFLC $(\kappa+\lambda)$ below the cut-off, $62(13.8 \%)$ had both abnormal sFLC $(\kappa / \lambda)$ and $\operatorname{sFLC}(\kappa+\lambda)$ above the cut-off with a concordance rate of $75.5 \%, 110$ cases were discordant $(24.5 \%)$ with 22 normal $\mathrm{sFLC}(\kappa / \lambda)$ and sFLC $(\kappa+\lambda)$ above the cut-off, and 88 with abnormal sFLC $(\kappa / \lambda)$ and sFLC $(\kappa+\lambda)$ below the cut-off. (C) Estimated Kaplan-Meier curves of TFS according to the combination of $\operatorname{SFCC}(\kappa / \lambda)$ abnormality and $\operatorname{sFLC}(\kappa+\lambda)$ cut-off value. To better illustrate the predictive impact of summated sFLC on TFS, separate curves for patients with normal $(D)$ or abnormal sFLC ratio $(E)$ are shown with respect to the $\operatorname{SFLC}(\kappa+\lambda)$ cut-off.

lymphocytes showing cytoplasmatic reactivity of either of the 2 FLCs, in accordance with the predominant clonal chain (Figure $5 \mathrm{~A}, \mathrm{D})$, a lower amount of lymphoid elements also showed partial reactivity of the nonclonal FLC (Figure 5B-C). These lymphocytes expressing different FLC types populated the same infiltrates in the lymph node and bone marrow and were associated with scattered FLC-producing plasma cells (Figure 5A-D insets). The specificity of the immunohistochemistry detection of FLCs was confirmed by the analysis of $\kappa$ and $\lambda$ expression in MM bone marrow infiltrates, in which FLC expression was almost entirely confined to the clone-related light chain (supplemental Figure 4). This preliminary demonstration of clone-related and unrelated light chain in the CLL microenvironment strengthens the rationale for assessment of $\operatorname{sFLC}(\kappa+\lambda)$.

\section{Discussion}

The management of CLL needs to be tailored to the expected disease course and to the patient's ability to tolerate treatments. An 
A

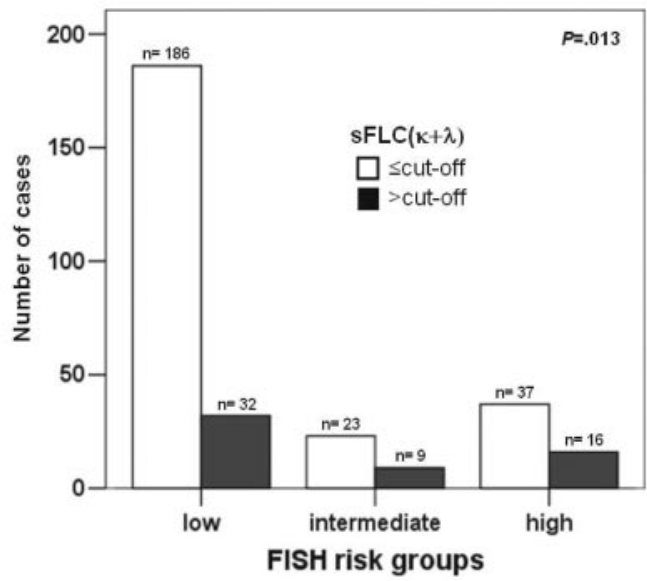

B
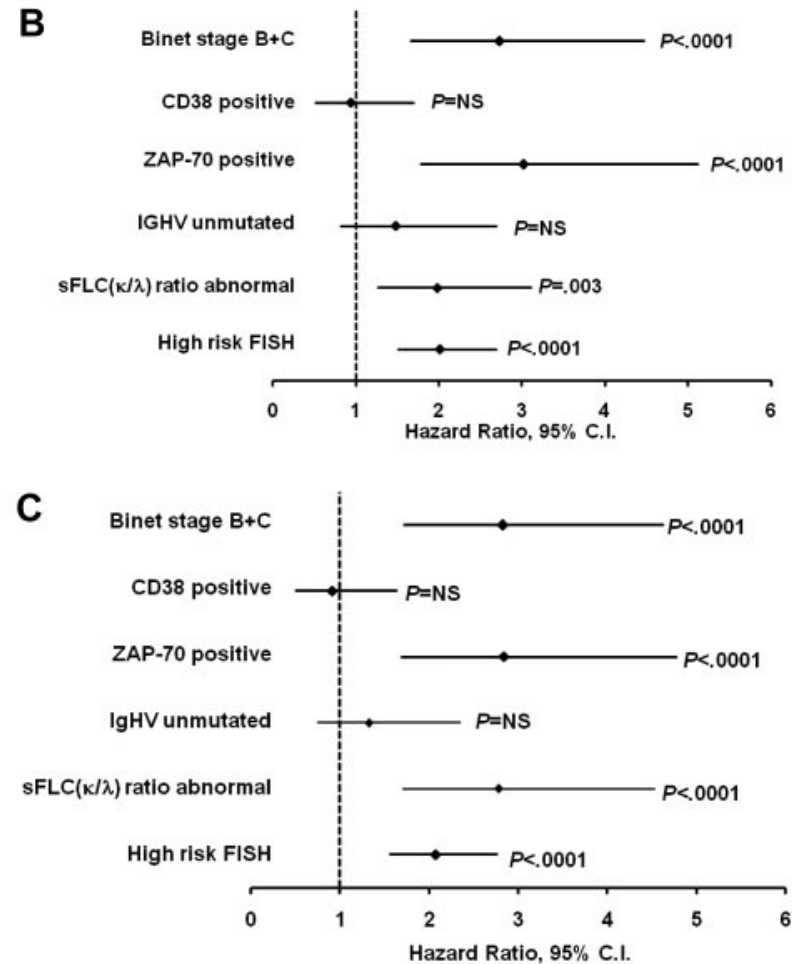

Figure 3. Comparison of cytogenetic risk and total sFLC. (A) CLL patients $(n=303)$, stratified by cytogenetic risk (low risk, normal and 13q14.3; intermediate risk, trisomy 12; high risk, 11q22.3 and 17p13.1) groups showed a significant correlation with $\mathrm{SFLC}(\mathrm{\kappa}+\lambda)$ cut-off value $(60.6 \mathrm{mg} / \mathrm{mL})$. Forest plots indicating (B) an abnormal sFLC $(\kappa / \lambda)(\mathrm{HR}=2.0,95 \% \mathrm{Cl}, 1.3-3.1)$ and $(\mathrm{C}) \mathrm{sFLC}(\kappa+\lambda)$ above the cut-off $(\mathrm{HR}=2.8,95 \% \mathrm{Cl}, 1.7-4.5)$ retained an independent association with TFS together with cytogenetics, ZAP-70 expression, and Binet stage in Cox multivariate models.

ideal prognostic biomarker, beyond being technically simple and cost-effective, should stratify untreated patients according to risk of progressive disease and shorter survival. This would allow identification of patients who may potentially benefit from early therapy and spare others unnecessary treatment-related toxicity.

Given its ability to accurately identify and monitor clonal B-cell populations, the sFLC assay has been explored as a potential new prognostic tool for CLL. ${ }^{7,8}$ Independent investigations have shown that $40 \%$ to $53 \%$ of CLL patients display an abnormal $\operatorname{sFLC}(\kappa / \lambda)$ and/or elevated levels of clonally unrestricted $\kappa$ or $\lambda$ sFLC, which have been proposed to identify cases with poor survival and early treatment requirement. ${ }^{7,8}$ Results of these retrospective studies are varied in terms of the prognostic value and independent impact of sFLC-dependent variables within the context of common CLL biomarkers. Pratt et al showed that $\operatorname{sFLC}(\kappa / \lambda)$ abnormalities predicted TFS if both treated and therapy-naive patients were grouped together; however, statistical significance did not hold if untreated patients were analyzed separately. ${ }^{7}$ Yegin et al reported that, although sFLC elevations identify patients requiring early treatment, median TFS did not statistically differ between cases with or without abnormal $\operatorname{sFLC}(\kappa / \lambda) .{ }^{8}$ Similarly, $\operatorname{sFLC}(\kappa / \lambda)$ abnormalities correlated with ZAP-70 but not with CD38 expression in the former study, ${ }^{7}$ whereas the opposite result was found in the latter. ${ }^{8}$ More importantly, sFLC levels and $\operatorname{sFLC}(\kappa / \lambda)$ abnormalities failed to stratify patients according to cytogenetic risk in one study, whereas FISH data were not reported in the other. ${ }^{7,8}$

By analyzing the largest reported cohort $(n=449)$ of untreated CLL patients, concurrently characterized for established CLL biomarkers, we document herein that $\operatorname{sFLC}(\kappa / \lambda)$ and sFLC levels are distinct prognostic variables in CLL. Although abnormal $\operatorname{sFLC}(\kappa / \lambda)$ was associated with parameters reflecting disease aggressiveness (ie, stage, CD38/ZAP-70, IGHV mutations, and independently predicted TFS), it did not correlate with cytogenetic risk. In contrast, unfavorable cytogenetics were associated with a novel biologic variable, namely, the $\operatorname{sFLC}(\kappa+\lambda)$, representing the sum of absolute $\kappa$ and $\lambda$ sFLC protein levels, irrespective of $\operatorname{sFLC}(\kappa / \lambda)$ abnormalities. Accordingly, we documented that the prognostic impact of an abnormal $\operatorname{sFLC}(\kappa / \lambda)$ becomes irrelevant if the $\operatorname{sFLC}(\kappa+\lambda)$ value is above a threshold (ie, $60.6 \mathrm{mg} / \mathrm{mL}$ ) derived from receiver operating characteristic analysis to discriminate patients who were actually treated from those never given therapy at the longest follow-up. Indeed, on multivariate analysis, $\mathrm{sFLC}(\kappa+\lambda)$ more than $60.6 \mathrm{mg} / \mathrm{mL}$ remained the strongest predictor of TFS together with ZAP-70, staging, and cytogenetics, whereas CD38, IGHV mutations, and $\operatorname{sFLC}(\kappa / \lambda)$ abnormalities lost

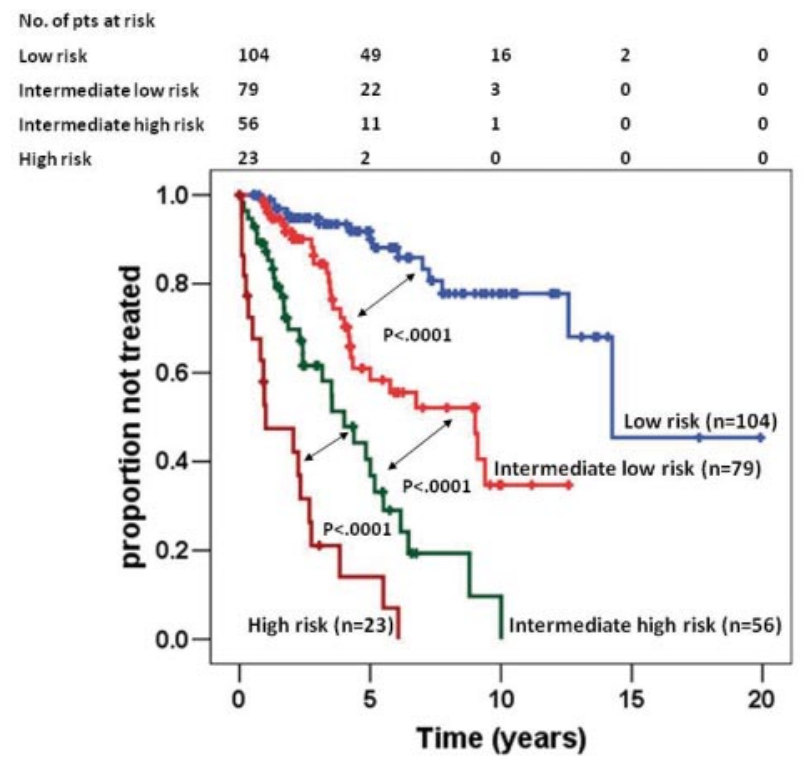

Figure 4. TFS of CLL patients according to a prognostic scoring system, including the $\operatorname{sLC}(\kappa+\lambda)$ parameter. Kaplan-Meier curves show the probability of remaining treatment-free for CLL cases subdivided according to a scoring system designed to evaluate unfavorable negative prognostic biomarkers: 1 point was assigned to each unfavorable prognostic marker [ZAP-70 expression, FISH abnormalities, Binet stage, and $\operatorname{sFCC}(\kappa+\lambda)]$, and the final score was given by the sum of the unfavorable markers present. A total of 262 patients were analyzed producing the following scores: 104 cases scored 0 (low risk), 79 scored 1 (low-intermediate risk), 56 scored 2 (intermediate-high risk), and 23 cases scored 3 or 4 (high risk) with a 3 -year TFS of $94.8 \%, 84.5 \%, 61.6 \%$, and $21.1 \%$, respectively. 
Figure 5. In situ immunohistochemical detection of free $\kappa$ and $\lambda$ chains in the CLL microenvironment. (A-B) In lymph node infiltrates of a $\mathrm{k}$-chain CLL case, a prominent population of plasmacytoid lymphocytes expressing the $\mathrm{\kappa} F \mathrm{LC}$ is detected along with a lower number of lymphocytes showing expression of the clone-unrelated $\lambda$ chain, both populating the same microenvironment. Scattered plasma cells with either $\kappa$ or $\lambda$ FLC expression are also detected (panels $A$ and $B$ insets). (C-D) A similar picture is observed in bone marrow lymphoid infiltrates of a $\lambda$-chain CLL case. Immunohistochemistry performed by the streptavidin-avidin-biotin complex using the 3-3'-diaminobenzidine chromogen (brown signal). Original magnifications $\times 200$ for all panels and $\times 400$ for insets.

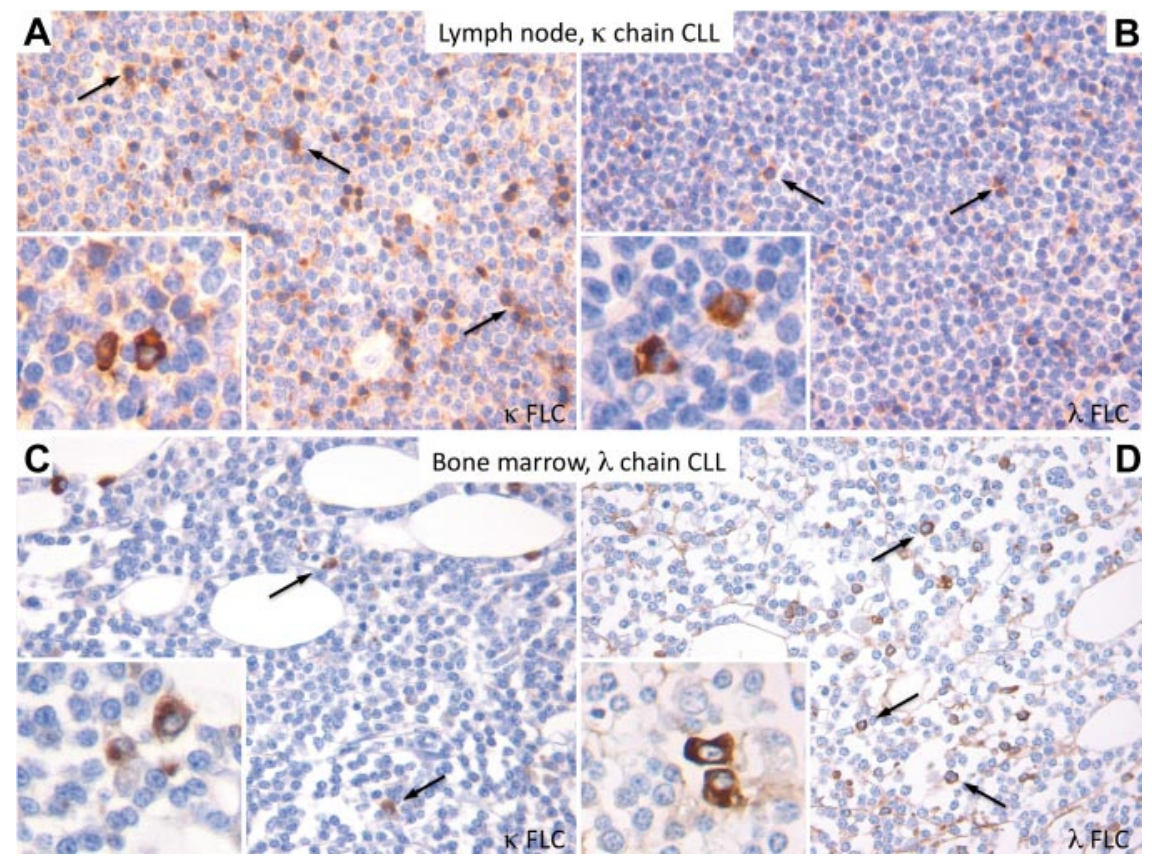

their prognostic power. A straightforward model, including sFLC $(\kappa+\lambda)$ more than $60.6 \mathrm{mg} / \mathrm{mL}$, Binet staging, ZAP-70, and cytogenetics, was validated to accurately predict time to treatment requirement in untreated CLL patients.

A high summated level of $\kappa$ plus $\lambda$ FLC could derive from the concurrence of cases with an excess monoclonal production (ie, with an abnormal FLC ratio) and cases with an excess polyclonal production (ie, with a normal FLC ratio), within the context of normal/defective kidney function. Even though CLL is not typically associated with renal injury, we excluded biases from non-tumor-related renal impairments by adopting adjusted sFLC normality ranges, in patients with impaired creatinine clearance. ${ }^{5} \mathrm{~A}$ separate analysis of patients with normal or abnormal FLC ratio indicated that a $\operatorname{sFLC}(\kappa+\lambda)$ above the cut-off retained its ability to predict TFS in both groups. A direct comparison evidenced that patients with a normal FLC $(\kappa / \lambda)$ and $\operatorname{sFLC}(\kappa+\lambda)$ above threshold displayed a poorer TFS outcome compared with all those with an sFLC $(\kappa+\lambda)$ below the cut-off, irrespective of ratio abnormalities. Interestingly, the outcome of patients concurrently displaying elevated $\operatorname{sFLC}(\kappa+\lambda)$ and abnormal $\operatorname{sFLC}(\kappa / \lambda)$ was not statistically different from those with $\operatorname{sFLC}(\kappa+\lambda)$ above the cut-off and a normal ratio. Both these cohorts of patients had the worst TFS. These data, beyond confirming our multivariate model, suggest that $\operatorname{sFLC}(\kappa / \lambda)$ and $\operatorname{sFLC}(\kappa+\lambda)$ reflect different phenomena in the complex biology of CLL.

Until recently, attention has been mostly focused on $\operatorname{sFLC}(\kappa / \lambda)$ and levels of "involved" (clonal) FLCs, which, being secreted by tumor cells, were thought to reflect size and biologic activity of the malignant B-cell population. ${ }^{2}$ Modern views maintain that CLL arises from activated, antigen-experienced $\mathrm{B}$ cells, suggesting that persistent immune stimulation and ongoing polyclonal B-cell activation/dysfunction may play an important role in the development and progression of this malignancy. ${ }^{22}$ Polyclonal B cells share the same microenvironmental interactions as tumor cells and could themselves be activated on antigenic stimulation of the CLL clone. ${ }^{23}$ This latter may in turn convey activatory signals to bystander B and T lymphocytes. ${ }^{24,25}$ In this scenario, tumor-derived monoclonal FLCs can be admixed with variable amounts of sFLC produced by nonclonal bystander B cells in lymphohematopoietic tissues. We documented a concurrence of these different B-cell populations to FLC synthesis within the CLL microenvironment. Our immunohistochemistry studies showed that tumor cells producing clonally restricted FLCs coexist with variable amounts of lymphocytes expressing nonclonal FLCs in infiltrated bone marrow and lymph nodes. These latter B cells might be in part clonally related to the dominant CLL clone and/or mirror an expanding antigen-stimulated polyconal B-cell pool. ${ }^{24}$ Interestingly, an ongoing B-cell activation/expansion, reflected by the overproduction of polyclonal sFLC, underlies the pathogenesis of several inflammatory/autoimmune conditions, which are themselves associated with an increased risk of non-Hodgkin lymphoma. ${ }^{26}$ Along this line, Tsai et al demonstrated that $38 \%$ of patients eventually developing CLL displayed an abnormal $\mathrm{sFLC}(\kappa / \lambda)$ up to 10 years before diagnosis and another $16 \%$ of them had a sustained polyclonal sFLC elevation in the same prediagnostic time frame. ${ }^{27}$ As such, elevations in both the "involved" sFLC (ie, the same clonally restricted light chain later found on tumor cells) and "uninvolved" FLCs can be found during pre-CLL phases. ${ }^{27}$ Similarly, it was shown that "low-count" monoclonal B-cell lymphocytosis, which shares many phenotypic traits of CLL, may actually be oligoclonal by reflecting the expansion of diversified, clonally related, populations with evidence of antigen drive. ${ }^{28}$ Finally, the presence of polyclonal sFLC elevations was shown to represent a strong risk factor for non-Hodgkin lymphoma development in HIV-infected persons. ${ }^{29}$ Although the mechanisms underlying polyclonal/oligoclonal FLC synthesis in "premalignant" CLL phases may reflect recruitment of multiple B-cell populations because of chronic antigenic challenge, the emergence of nonclonal FLCs at an overt tumor stage, as suggested by our study, is more puzzling. The coexistence of clone "side populations," with a diversified light-chain repertoire resulting from aberrant recombinase activity, could be hypothesized given that Ig recombination also occurs in mature B-cell populations, on persistent autoantigenic stimulation, to preserve/restore tolerance. ${ }^{30-33}$ Thus, molecular events involving the neoplastic clone and the environmental stimuli sustaining bystander B-cell 
activation may both concur to the sFLC pattern of CLL and to its prognostic relevance.

The overproduction of nonclonal sFLC in CLL patients may not necessarily be related to tumor biology. Results of populationbased studies have indicated that overproduction of nonclonal sFLC could predict mortality in individuals older than 50 years, who were either healthy or affected by various comorbidities. ${ }^{34}$ Based on these findings, polyclonal sFLC elevation was interpreted as a nonspecific marker of "unwellness," which reflects a generalized immune overstimulation occurring in elderly subjects. $^{34}$

Contextualizing this view to our cohort of aged CLL patients would, however, argue in favor of the highly significant association of the $\operatorname{sFLC}(\kappa+\lambda)$ variable with intrinsic features of tumor cells, such as ZAP-70 and unfavorable cytogenetics, and its strong and independent predictive power on TFS. Although host "fitness" may certainly affect overall survival of CLL patients, its direct influence on time to treatment requirement, an outcome mostly related to tumor biologic aggressiveness itself, is more complex to demonstrate.

Independently of the mechanisms underlying the overproduction of sFLCs, these can "per se" endorse immunobiologic activities relevant to CLL pathobiology. For instance, despite being usually regarded as a mere spillover product of Ig synthesis/assembly, FLCs could activate mast cells, which enrich the CLL microenvironment. ${ }^{35}$ Activated mast cells may in turn support tumor cell growth and foster immune stimulation through different pathways, including CD40- and IL-6mediated signaling. ${ }^{36}$

In conclusion, our findings support that the cumulative amount of circulating FLC, irrespective of their clonality, is a strong and independent prognostic predictor in CLL. A new scoring system, involving the simple, reproducible, and cost-effective sFLC assay, is proposed to identify at diagnosis CLL patients who are more likely to require early treatment.

\section{Acknowledgments}

The authors thank Dr Alessandro Marchei, The Binding Site, Italy, for help and advice on FLC testing, and Prof Alan Solomon, Human Immunology \& Cancer Program, University of Tennessee Graduate School of Medicine, for providing the anti- $\kappa$ and anti- $\lambda$ FLC monoclonal antibodies used in the immunohistochemistry studies.

This work was supported by Associazione Italiana Ricerca sul Cancro (AN-IG4569, MF-IG10492, and FM-RG6432), Associazione Italiana Ricerca sul Cancro-Special Program Molecular Clinical Oncology (5 per mille, grant 9980, 2010-15; A.N., M.F., and F.M.) Ricerca Finalizzata from Italian Ministry of Health 2006 (G.C., F.M., and M.F.) and 2007 (G.C.), Fondo Investimento per la Ricerca de Base (grant RBIP06LCA9; M.F.), progetto Compagnia San Paolo (G.C.), the Ministero della Salute, Ricerca Corrente, and Ricerca Finalizzata FSN, Rome, Italy (A.P.). V.G. was supported by a fellowship from Associazione Italiana Ricerca sul Cancro.

\section{Authorship}

Contribution: F.M., R.D.F., and A.P. designed the study, analyzed the data, and wrote the manuscript; R.C. and V.G. performed assays; G.C., A.N., and G.D.P. performed biomarker analysis; B.A., A.G.R., M.F., and C.T. analyzed data and reviewed the manuscript; L.L., K.Z., M.G., E.V., S.M., G.D.P., and E.M. provided material; and all authors approved the final version of the manuscript.

Conflict-of-interest disclosure: B.A. is the Medical Director at The Binding Site Ltd, Italy. The remaining authors declare no competing financial interests.

Correspondence: Fortunato Morabito, Unità Operativa Complessa di Ematologia, Dipartimento di Onco-Ematologia, Azienda Ospedaliera di Cosenza, Viale della Repubblica, 87100 Cosenza, Italy; e-mail: fortunato_morabito@tin.it.

\section{References}

1. Katzmann JA, Clark RJ, Abraham RS, et al. Serum reference intervals and diagnostic ranges for free kappa and free lambda immunoglobulin light chains: relative sensitivity for detection of monoclonal light chains. Clin Chem. 2002;48(9):14371444.

2. Bradwell AR, Carr-Smith HD, Mead GP, et al. Highly sensitive, automated immunoassay for immunoglobulin free light chains in serum and urine. Clin Chem. 2001;47(4):673-680.

3. Dispenzieri A, Kyle R, Merlini G, et al. International Myeloma Working Group guidelines for serum-free light chain analysis in multiple myeloma and related disorders. Leukemia. 2009;23(2):215224.

4. Thio M, Blokhuis BR, Nijkamp FP, Redegeld FA. Free immunoglobulin light chains: a novel target in the therapy of inflammatory diseases. Trends Pharmacol Sci. 2008;29(4):170-174.

5. Hutchison CA, Harding S, Hewins P, et al. Quantitative assessment of serum and urinary polyclonal free light chains in patients with chronic kidney disease. Clin J Am Soc Nephrol. 2008; 3(6):1684-1690.

6. Gordon J, Howlett AR, Smith JL. Free light chain synthesis by neoplastic cells in chronic lymphocytic leukaemia and non-Hodgkin's lymphoma. Immunology. 1978;34(3):397-404.

7. Pratt G, Harding S, Holder R, et al. Abnormal serum free light chain ratios are associated with poor survival and may reflect biological subgroups in patients with chronic lymphocytic leukaemia. Br J Haematol. 2009;144(2):217-222.

8. Yegin ZA, Ozkurt ZN, Yagci M. Free light chain: a novel predictor of adverse outcome in chronic lymphocytic leukemia. Eur J Haematol. 2010; 84(5):406-411.

9. Zenz T, Frohling S, Mertens D, Dohner H, Stilgenbauer $\mathrm{S}$. Moving from prognostic to predictive factors in chronic lymphocytic leukaemia (CLL). Best Pract Res Clin Haematol. 2010;23(1):71-84.

10. Hallek M, Cheson BD, Catovsky D, et al. Guidelines for the diagnosis and treatment of chronic lymphocytic leukemia: a report from the International Workshop on Chronic Lymphocytic Leukemia updating the National Cancer Institute-Working Group 1996 guidelines. Blood. 2008;111(12): 5446-5456.

11. Matutes E, Owusu-Ankomah K, Morilla R, et al. The immunological profile of B-cell disorders and proposal of a scoring system for the diagnosis of CLL. Leukemia. 1994;8(10):1640-1645.

12. Cutrona G, Colombo M, Matis S, et al. Clonal heterogeneity in chronic lymphocytic leukemia cells: superior response to surface IgM cross-linking in CD38, ZAP-70-positive cells. Haematologica. 2008;93(3):413-422.

13. O'Brien S, Keating MJ. Chronic lymphoid leukemias. In: De Vita T, Hellman J, Rosenberg S, eds. Cancer Principle \& Practice of Oncology. Vol 2.
Philadelphia, PA: Lippincott Williams \& Wilkins; 2005:2133-2142.

14. Del Poeta G, Maurillo L, Venditti A, et al. Clinical significance of CD38 expression in chronic lymphocytic leukemia. Blood. 2001;98(9):2633-2639.

15. Del Principe MI, Del Poeta G, Buccisano F, et al. Clinical significance of ZAP-70 protein expression in B-cell chronic lymphocytic leukemia. Blood. 2006;108(3):853-861.

16. Morabito F, Cutrona G, Gentile M, et al. Definition of progression risk based on combinations of cellular and molecular markers in patients with Binet stage A chronic lymphocytic leukaemia. $\mathrm{Br} J$ Haematol. 2009;146(1):44-53.

17. Fais F, Ghiotto F, Hashimoto S, et al. Chronic lymphocytic leukemia $B$ cells express restricted sets of mutated and unmutated antigen receptors. J Clin Invest. 1998;102(8):1515-1525.

18. Fabris S, Mosca L, Todoerti K, et al. Molecular and transcriptional characterization of 17p loss in B-cell chronic lymphocytic leukemia. Genes Chromosomes Cancer. 2008;47(9):781-793.

19. Tripodo C, Gri G, Piccaluga PP, et al. Mast cells and Th17 cells contribute to the lymphoma-associated pro-inflammatory microenvironment of angioimmunoblastic T-cell lymphoma. Am J Pathol. 2010;177(2):792-802.

20. Altman D. Practical Statistics for Medical Research. London, United Kingdom: Chapman Hall; 1991. 
21. Harrell FE Jr, Lee KL, Mark DB. Multivariable prognostic models: issues in developing models, evaluating assumptions and adequacy, and measuring and reducing errors. Stat Med. 1996;15(4): 361-387.

22. Kostareli E, Sutton LA, Hadzidimitriou A, et al. Intraclonal diversification of immunoglobulin light chains in a subset of chronic lymphocytic leukemia alludes to antigen-driven clonal evolution. Leukemia. 2010;24(7):1317-1324.

23. Ayala F, Dewar R, Kieran M, Kalluri R. Contribution of bone microenvironment to leukemogenesis and leukemia progression. Leukemia. 2009; 23(12):2233-2241

24. Van den Hove LE, Van Gool SW, Vandenberghe $\mathrm{P}$, et al. CD40 triggering of chronic lymphocytic leukemia B cells results in efficient alloantigen presentation and cytotoxic $T$ lymphocyte induction by up-regulation of CD80 and CD86 costimulatory molecules. Leukemia. 1997;11(4):572-580.

25. Lapalombella R, Andritsos L, Liu Q, et al. Lenalidomide treatment promotes CD154 expression on CLL cells and enhances production of antibodies by normal B cells through a PI3-kinase- dependent pathway. Blood. 2010;115(13):26192629.

26. Gottenberg JE, Aucouturier F, Goetz J, et al. Serum immunoglobulin free light chain assessment in rheumatoid arthritis and primary Sjogren's syndrome. Ann Rheum Dis. 2007;66(1):23-27.

27. Tsai HT, Caporaso NE, Kyle RA, et al. Evidence of serum immunoglobulin abnormalities up to 9.8 years before diagnosis of chronic lymphocytic leukemia: a prospective study. Blood. 2009; 114(24):4928-4932.

28. Lanasa MC, Allgood SD, Volkheimer AD, et al. Single-cell analysis reveals oligoclonality among 'low-count' monoclonal B-cell lymphocytosis. Leukemia. 2010;24(1):133-140.

29. Landgren O, Goedert JJ, Rabkin CS, et al. Circulating serum free light chains as predictive markers of AIDS-related lymphoma. J Clin Oncol. 2010;28(5):773-779.

30. Oltz EM. Regulation of antigen receptor gene assembly in lymphocytes. Immunol Res. 2001;23(23):121-133.

31. Rice JS, Newman J, Wang C, Michael DJ, Diamond B. Receptor editing in peripheral B cell tol- erance. Proc Natl Acad Sci U S A. 2005;102(5): 1608-1613.

32. Labrie JE 3rd, Sah AP, Allman DM, Cancro MP, Gerstein RM. Bone marrow microenvironmental changes underlie reduced RAG-mediated recombination and $B$ cell generation in aged mice. $J$ Exp Med. 2004;200(4):411-423.

33. Saborit-Villarroya I, Vaisitti T, Rossi D, et al. E2A is a transcriptional regulator of CD38 expression in chronic lymphocytic leukemia. Leukemia. 2011; 25(3):479-488.

34. Dispenzieri A, Katzmann JA, Kyle RA, Larson D, Rajkumar SV. Non-clonal serum immunoglobulin free light chains (FLC) as markers of overall survival. Hematol Rep. 2010;2(s2):12.

35. Rijnierse A, Redegeld FA, Blokhuis BR, et al. Igfree light chains play a crucial role in murine mast cell-dependent colitis and are associated with human inflammatory bowel diseases. $J$ Immunol. 2010;185(1):653-659.

36. Tripodo C, Sangaletti S, Piccaluga PP, et al. The bone marrow stroma in hematological neoplasmas- a guilty bystander. Nat Rev Clin Oncol. 2011;8(8):456-466. 